\title{
Regulation of neuroinflammation through programed death-1/programed death ligand signaling in neurological disorders
}

\author{
Shangfeng Zhao ${ }^{1,2}$, Fengwu Li ${ }^{3}$, Rehana K. Leak ${ }^{4}$, Jun Chen ${ }^{1}$ and Xiaoming Hu ${ }^{1,3}$ * \\ 1 Department of Neurology, University of Pittsburgh School of Medicine, Pittsburgh, PA, USA \\ ${ }^{2}$ Department of Neurosurgery, Beijing Tongren Hospital, Capital Medical University, Beijing, China \\ ${ }^{3}$ Institute of Neuroscience, Luhe Hospital, Capital Medical University, Beijing, China \\ ${ }^{4}$ Division of Pharmaceutical Sciences, Duquesne University, Pittsburgh, PA, USA
}

Edited by:

Arthur Liesz, University Hospital Munich, Germany

\section{Reviewed by:}

Vikramjeet Singh, Ludwig-

Maximilians-Universität, Germany Halina Offner, Oregon Health \&

Science University, USA

${ }^{*}$ Correspondence:

Xiaoming Hu, Department of

Neurology, University of Pittsburgh,

3500 Terrace Street, Pittsburgh, PA

15213, USA

e-mail:hux2@upmc.edu
Immune responses in the central nervous system (CNS), which involve both resident glial cells and infiltrating peripheral immune cells, play critical roles in the progress of brain injuries and neurodegeneration. To avoid inflammatory damage to the compromised brain, the immune cell activities in the CNS are controlled by a plethora of chemical mediators and signal transduction cascades, such as inhibitory signaling through programed death-1 (PD-1) and programed death ligand (PD-L) interactions. An increasing number of recent studies have highlighted the importance of PD-1/PD-L pathway in immune regulation in CNS disorders such as ischemic stroke, multiple sclerosis, and Alzheimer's disease. Here, we review the current knowledge of the impact of PD-1/PD-L signaling on brain injury and neurodegeneration. An improved understanding of the function of PD-1/PD-L in the cross-talk between peripheral immune cells, CNS glial cells, and non-immune CNS cells is expected to shed further light on immunomodulation and help develop effective and safe immunotherapies for CNS disorders.

Keywords: PD-1, PD-L1, stroke, neurodegeneration, inflammation

\section{INTRODUCTION}

The central nervous system (CNS) was traditionally thought to tolerate the invasion of antigens without an inflammatory response. The presence of an intact blood-brain barrier (BBB) and the lack of lymphatic vessels in the brain was believed to restrict the infiltration of peripheral immune cells into brain parenchyma under physiological conditions and maintain the CNS in a so-called "immune-privileged" state (Engelhardt, 2008). Recent research, however, has resulted in a revision of this concept. Compelling data now suggest that the CNS is actually immunocompetent and not completely immune-privileged. A series of neuroinflammatory responses, involving both resident CNS glial cells and peripheral immune cells invading via the damaged $\mathrm{BBB}$, are promptly launched in response to noxious stimuli (Zipp and Aktas, 2006). These immune cells are important for defense against CNS infection, injury, or neurodegeneration and for CNS repair and regeneration. Their activities, however, have to be carefully regulated to avoid inflammatory damage to already compromised and highly vulnerable tissues of the CNS.

The tendency of the immune system to damage bystander tissue is kept in check by a series of self-regulating, inhibitory systems that preserve immune homeostasis. For example, inhibitory signaling through programed death-1 (PD-1) and programed death ligand (PD-L) interactions is an important mechanism underlying immune regulation in many pathological circumstances, such as autoimmune diseases, cancer, and organ transplantation. Recent evidence indicates that the PD-1/PD-L system is also critical in reducing the inflammatory responses in CNS diseases such as stroke, multiple sclerosis (MS), and Alzheimer's disease (AD) (Kroner et al., 2005; Ren et al., 2011b; Saresella et al., 2012). Here, we review our accumulated understanding of the PD-1/PD$\mathrm{L}$ pathway, with a special emphasis on its potential role in brain injuries and neurodegenerative diseases.

\section{FUNCTIONS OF PD-1/PD-L1 IN IMMUNE RESPONSES}

Programed death-1 (or CD279) is a $50-55 \mathrm{kDa}$ member of the CD28 family of T-cell regulators (Riley and June, 2005). It is expressed at a low level on naïve T-cells and can be induced upon activation in many types of immune cells, including Tcells, B cells, natural killer (NK) cells, monocytes, and dendritic cells (DCs). Structurally, PD-1 is composed of an N-terminal IgV-like domain, an approximately 20 amino acid-long stalk, a transmembrane domain, and a cytoplasmic domain. The cytoplasmic domain contains two tyrosine-based signaling motifs: an immunoreceptor tyrosine-based inhibitory motif (ITIM) and an immunoreceptor tyrosine-based switch motif (ITSM), both of which are essential for PD-1 function (Zhang et al., 2004). PD-1 has two binding ligands: PD-L1 (CD274) and PD-L2 (CD273). Both of these ligands are members of the B7 family of costimulatory molecules. PD-L1 is broadly expressed on a variety of hematopoietic cells (including T-cells, B cells, DCs, and monocytes) in addition to many non-hematopoietic cells, such as epithelial and endothelial cells. In contrast, the expression of PD-L2 is mainly restricted to antigen presenting cells (APCs), including macrophages, DCs, and specific B cell subpopulations (Zhong et al., 2007). In general, PD-L2 is expressed 
at lower levels but binds to PD-1 with higher affinity than PDL1 (Ghiotto et al., 2010).

The breadth of expression of $\mathrm{PD}-1$ and $\mathrm{PD}-\mathrm{L}$ in multiple types of immune cells suggests a wide range of functions in immunomodulation. First and foremost, the main role of PD$1 / \mathrm{PD}-\mathrm{L} 1$ is to act as a negative regulatory system to fine-tune T-cell and $\mathrm{B}$ cell activity. The engagement of T or B cell-expressed PD-1 with PD-L on APCs relays inhibitory signals that down-regulate T-cell receptor (TCR) or B cell receptor (BCR)-mediated cell activation (Freeman et al., 2000; Latchman et al., 2001; Okazaki et al., 2001; Yokosuka et al., 2012). As a consequence of this interaction and downstream effect, the PD-1/PD-L system plays critical roles in many $\mathrm{T}$ or $\mathrm{B}$ cell-mediated immune responses, including immunity to infection, antibody production, immune tolerance, and autoimmunity (Okazaki et al., 2013). For example, mounting evidence reveals the importance of the $\mathrm{PD}-1 / \mathrm{PD}-\mathrm{L}$ pathway in the maintenance of central and peripheral tolerance. On the one hand, PD-1/PD-L1 interactions regulate T-cell selection and shape Tcell repertoires in the thymus (Blank et al., 2003; Keir et al., 2005). Absence of PD-1 alters the signaling threshold during T-cell development in thymus and leads to increased emergence of CD4/CD8 double-negative $\alpha \beta$ T-cells. On the other hand, the PD-1/PD-L1 pathway also induces T-cell tolerance and inhibits self-reactive Tcell proliferation and cytokine production in peripheral lymph organs or tissues (Probst et al., 2005). Deficiency of PD-1 or blockade of PD-1/PD-L signaling results in the development or exacerbation of autoimmune diseases in mouse models of lupuslike glomerulonephritis/arthritis, cardiomyopathy, type I diabetes, experimental autoimmune encephalomyelitis (EAE), and autoimmune enteritis (Nishimura et al., 1999, 2001; Salama et al., 2003; Fife et al., 2009; Reynoso et al., 2009). These findings suggest that PD-1/PD-L signaling plays an important protective role against multiple types of autoimmune disorders. Interestingly, there is some variation in the autoimmune disease phenotype depending on the genetic background of different mouse stains, indicating perhaps that lymphocyte regulation through $\mathrm{PD}-1 / \mathrm{PD}-\mathrm{L}$ is highly antigen specific (Okazaki et al., 2013).

Recent studies reveal that the PD-1/PD-L interaction also regulates the functions of cells other than lymphocytes through multiple mechanisms. First, the PD-1/PD-L1 interaction between T-cells and APCs may be bidirectional, enabling some degree of reciprocal communication. For example, PD-1 on T-cells activates PD-L1 on macrophages and induces a regulatory macrophage profile with enhanced IL-10 and reduced IL-6 production (Lee et al., 2013). Second, PD-1 signaling may function in APCs independently of TCR or BCR activation. One example is that the ligation of monocyte PD-1 with PD-L1 directly stimulates IL-10 production, leading to reversible $\mathrm{CD} 4^{+} \mathrm{T}$-cell dysfunction after HIV infection (Said et al., 2010). In addition, cross-linking of PD-L2 on DCs with specific IgM directly stimulates DC functions and activates groups of genes involved in cell migration and survival (Blocki et al., 2006). These studies demonstrate that PD-1/PD-L signaling blunts immune overreaction and prevents cellular toxicity.

Unfortunately, our current knowledge about PD-1/PD-L functions in different immune cells is still limited. Further exploration of this field is expected to extend our understanding of the impact of this inhibitory signaling system and evaluate its therapeutic potential in immune-related diseases.

\section{PD-1/PD-L1 SIGNALING PATHWAY}

PD-1/PD-L1 signaling has been studied most extensively in $\mathrm{T}$ and $\mathrm{B}$ lymphocytes. In these cells, $\mathrm{PD}-1$ ligation induces signal transduction only when there is simultaneous activation of BCR or TCR. The binding of PD-1 with PD-L1, along with antigen recognition, results in the phosphorylation of tyrosine residues in the ITSM and subsequent recruitment of SH2 domain-containing phosphatase-2 (SHP-2), or less frequently, SHP-1 (Freeman et al., 2000; Latchman et al., 2001; Okazaki et al., 2001; Yokosuka et al., 2012). SHP-2 and SHP-1 are two highly related tyrosine phosphatases that dephosphorylate proximal signaling molecules such as Syk downstream of BCR or Zap70 downstream of TCR (Okazaki et al., 2001; Sheppard et al., 2004). This dephosphorylation attenuates the signaling cascades engaged by antigen recognition and diminishes the ensuing biological effects. The PD-1/PD-L1 activated signaling pathways in other types of cells remain to be characterized.

\section{PD-1 AND PD-L1 IN ISCHEMIC STROKE}

Stroke is an acute brain injury closely associated with strong and persistent inflammation. Post-stroke inflammation is characterized by the activation of local microglia and the rapid accumulation of peripheral immune cells in the ischemic brain (Iadecola and Anrather, 2011). The antigen-non-specific immune responses mediated by innate immune cells (microglia, macrophage, neutrophil, NK cells, etc.) commence very early after stroke. In contrast, the lymphocyte-mediated adaptive immune responses become prominent at later stages (since 3-4 days after onset) of stroke, although these lymphocytes may migrate into the ischemic boundary within the first $24 \mathrm{~h}$ of reperfusion (Gelderblom et al., 2009). Mounting evidence demonstrates that lymphocytes play pivotal roles in both brain injury and brain recovery. For instance, deficiency of either $\mathrm{CD}^{+}{ }^{+}$or $\mathrm{CD} 8^{+} \mathrm{T}$-cells resulted in the reduction in infarct volume and improvement in neurological performance in experimental models of stroke, suggesting detrimental roles of $\mathrm{CD}^{+}$and $\mathrm{CD}^{+}$T-cells after stroke (Yilmaz et al., 2006; Liesz et al., 2011). However, some specific lymphocyte populations, including regulating $\mathrm{T}$-cells and regulating $\mathrm{B}$ cells, have been shown to be protective to ischemic brain or promote brain recovery after stroke (Liesz et al., 2009; Li et al., 2013). Further elucidating the mechanisms underlying intricate immunoregulation after stroke is critical not only for basic research of the immune system and CNS injury but also for the clinical translation of new therapeutic candidates.

Several recent publications have highlighted the importance of PD-1 and PD-L1 signaling in post-stroke inflammation and brain injury (Figure 1). For example, it has been reported that the expression of PD-L1 and PD-L2 on peripheral B cells is significantly increased 4 days after transient middle cerebral artery occlusion (MCAO), an established experimental model of stroke (Ren et al., 2011b). In the meantime, the expression of PD-1 is elevated on activated resident microglia and on infiltrating macrophages. These elevations in PD-1/PD-L support the notion that this coinhibitory pathway is intimately involved in the regulation of 


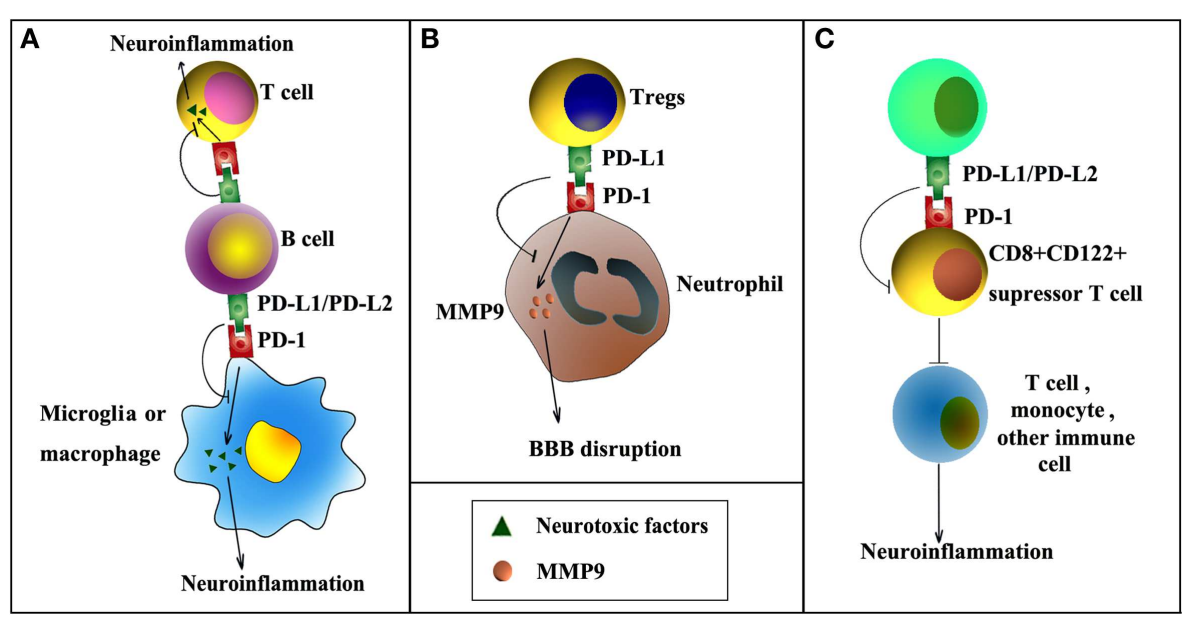

FIGURE 1 | PD-1/PD-L signaling in ischemic stroke. PD-1/PD-L signaling may influence post-stroke inflammation and functional outcomes by negatively regulating the following cell-cell interactions. (A) PD-L1/PD-L2 expression on B cells inhibits the activation of microglia, macrophages, or effector T-cells, thereby reducing inflammation in the ischemic brain. (B) PD-L1 expression on regulatory T-cells (Tregs) inhibits neutrophil-derived matrix metalloproteinase-9 (MMP-9) through PD-L1-PD-1 interactions and reduces subsequent blood-brain barrier (BBB) damage in the acute phase after stroke. (C) PD-L1/PD-L2 inhibits immunoregulatory CD8 ${ }^{+} \mathrm{CD} 122^{+}$ suppressor T-cells, reducing their recruitment into the CNS from the spleen after stroke. As a result, post-stroke inflammation and brain injury are enhanced. ischemic brain injury. Interestingly, experiments using PD-1 or PD-L1 knockout mice have shown diametrically opposed results. As would be expected from a protective role for PD-1, deficiency in PD-1 enlarges brain infarct sizes and exacerbates neurological deficits at 4 days after MCAO, and these events are accompanied by increased infiltration of $\mathrm{CD}^{+}{ }^{+} \mathrm{T}$-cells, $\mathrm{Gr} 1^{+}$neutrophils, macrophages, and exaggerated microglial activation (Ren et al., 2011b). In contrast, another study from the same research group using PD-L1 or PD-L2 knockout mice revealed that PD-L exacerbates post-stroke inflammation and plays a detrimental role in stroke outcomes (Bodhankar et al., 2013b). Mechanistically, the protective effects of PD-1 are attributed to its expression on $B$ cells and subsequent inhibition of inflammatory responses in other immune effector cells (Ren et al., 2011b). The detrimental effects of PD-L1, however, may depend on its inhibition of the recruitment of immunoregulatory $\mathrm{CD} 8^{+} \mathrm{CD} 122^{+}$suppressor T-cells from the spleen into the ischemic brain (Bodhankar et al., 2013b). $\mathrm{CD}^{+} \mathrm{CD} 122^{+}$regulatory T-cells are known to regulate other $\mathrm{CD}^{+} \mathrm{CD}_{122}-$ T-cells, which cause tissue damage when over-activated (Rifa'i et al., 2004). Thus, PD-L1 may release $\mathrm{CD}^{+}{ }^{+} \mathrm{CD} 122^{-}$T-cells from inhibition and thereby elicit injury. The opposing nature of PD-1 and PD-L in these studies may reflect the frequently dualistic nature of the immune system. Future immunotherapies will therefore have to account for the general complexity of immunomodulation in the injured brain.

A potential caveat of research on $\mathrm{PD}-1 / \mathrm{PD}-\mathrm{L}$ is worth discussing here. All the above-mentioned studies on PD-1 and PD-L in stroke rely on global gene knockout mice. Although these results provide valuable information about the overall effects of PD-1 and PD-L on stroke outcome, they are by themselves not sufficient to define the cell-specific functions of PD-1 and PD-L in stroke. In this regard, further studies using transgenic mice with cell-specific gene manipulations are necessary. In vitro studies are also warranted to confirm direct cell-cell interactions. One recent study from our group demonstrated direct interactions between regulatory T-cells (Tregs) and neutrophils through PDL1 and PD-1 (Li et al., 2014). This interaction was found to be essential for Treg-mediated suppression of neutrophil-derived matrix metalloproteinase-9 (MMP-9). In view of the importance of MMP-9 in early BBB disruption after stroke (Asahi et al., 2001; Rosell et al., 2006), we further showed that PD-L1 expression on Tregs mediates Treg-afforded neuroprotection against experimental stroke through the inhibition of peripheral neutrophil-derived MMP-9 and through subsequent preservation of BBB integrity (Li et al., 2014). In addition, another study demonstrated that adoptive transfer of IL-10-producing B cells into the stroke mice could increase the expression of PD-1 in peripheral CD4 ${ }^{+}$T-cells (Bodhankar et al., 2013a), suggesting that PD-1/PD-L1 interaction might also be important in regulatory $\mathrm{B}$ cell-provided neuroprotection after stroke (Ren et al., 2011a; Bodhankar et al., 2013a).

Thus far, all the research on PD-1/PD-L in stroke has focused on their short-term effects. Given the persistent immune responses after stroke and their contribution to brain recovery, the long-term influence of these inhibitory molecules in the ischemic brain is an important direction to pursue in the future.

\section{PD-1/PD-L1 PATHWAY IN NEURODEGENERATIVE DISEASES ALZHEIMER'S DISEASE}

Alzheimer's disease is an age-related neurodegenerative disease characterized by memory loss, progressive cognitive impairment, and neuropsychiatric disturbances. The pathological hallmarks of $\mathrm{AD}$ are the extracellular accumulation of amyloid plaques and intracellular deposition of neurofibrillary tangles (Liu et al., 2013). A $\beta$ accumulation in amyloid plaques leads to chronic 
neuroinflammation in the brain, thereby contributing to disease progression and poor functional outcomes (Rubio-Perez and Morillas-Ruiz, 2012). A reduction in suppressor cell function in the periphery has been observed in $\mathrm{AD}$ patients, as manifested by loss of balance in immune cell populations and decreased IL-10 production in the blood (Guerreiro et al., 2007; Speciale et al., 2007). Therefore, activation of immunoregulatory mechanisms during the progression of AD might be able to re-establish immune homeostasis.

Expression of PD-1 on CD4 ${ }^{+}$T-cells and PD-L1 on $\mathrm{CD} 14^{+}$ monocyte/macrophage significantly decrease in $\mathrm{AD}$ patients and patients with mild cognitive impairment (MCI), underscoring the importance of these molecules in AD (Saresella et al., 2012). Impairments in PD-1/PD-L1 are associated with inhibition of IL-10 production, suggesting an effect of this signaling system in boosting IL-10 production. IL-10 has been shown to limit inflammatory responses and ameliorate $\mathrm{AD}$ pathology in animal models (Koronyo-Hamaoui et al., 2009). A recent study showed that although the IL-10 serum levels are comparable in $\mathrm{AD}$ patients and healthy controls, the frequency of $\mathrm{CD} 4^{+} \mathrm{T}$ cells expressing IL-10 in AD group is much higher than that in controls, indication a systemic effort to counterbalance the proinflammatory responses in the AD brain (Torres et al., 2013). Thus, it is conceivable that a decrease in this protective cytokine in $\mathrm{AD}$ patients synergizes with an increased activity in $\mathrm{A} \beta$-reactive T-cells, thereby enhancing neuroinflammation and exacerbating brain pathology. In addition, the PD-1/PD-L1 interaction is shown to induce the apoptosis in $\mathrm{A} \beta$-specific $\mathrm{CD} 4^{+} \mathrm{T}$-cells (Saresella et al., 2012).

The expression of PD-1 on Tregs is also affected by AD pathology (Saresella et al., 2010). The number of PD-1 ${ }^{+}$Tregs is increased both in patients with fully developed $\mathrm{AD}$ and with MCI. In contrast, $\mathrm{PD}-1^{-}$Tregs are significantly increased only in MCI patients, but not in full-blown AD patients. Although $\mathrm{PD}-1$ has been known to promote Treg differentiation (Wang et al., 2010), the functional differences between PD- $1^{-}$Tregs and PD-1 ${ }^{+}$Tregs are not clear. Therefore, the significance of altered PD-1 expression on Tregs in $\mathrm{AD}$ and $\mathrm{MCI}$ patients awaits further investigation.

To date, our knowledge of the function of PD-1/PD-L1 in the pathology of $\mathrm{AD}$ is very limited. Further work is necessary to elucidate the cellular and molecular mechanisms of PD-1 or PD-L1 actions in $\mathrm{AD}$, such as their contribution to immune cell cross-talk in the CNS.

\section{MULTIPLE SCLEROSIS}

Multiple sclerosis is a chronic inflammatory neurodegenerative disease characterized by the demyelination of white matter and focal infiltration of immune cells in the CNS (Minagar et al., 2004; Compston and Coles, 2008). The CD4 ${ }^{+}$effector T-cells have long been considered as the most important infiltrating cells in MS. The involvement of other T-cell subtypes (interleukin-17-producing T-cells, CD8 ${ }^{+}$T-cells, Tregs, and $\gamma / \varphi$ T-cells), APCs, and microglia has also been supported (Viglietta et al., 2004; Langrish et al., 2005; Tzartos et al., 2008).

Mounting evidence highlights the importance of PD-1 and PD$\mathrm{L}$ in MS. The expression of PD-1 is significantly increased on myelin basic protein (MBP)-stimulated $\mathrm{CD}^{+}{ }^{+}$and $\mathrm{CD} 8^{+} \mathrm{T}$ lymphocytes isolated from the peripheral blood of patients with stable MS compared to lymphocytes from patients with acute remissions and relapses. Correspondingly, PD-L1-expressing APCs are increased in stable MS patients. Up-regulation of PD-1/PD-L1 enhances the apoptosis of MBP-specific cells, which is associated with disease remission in MS patients (Trabattoni et al., 2009). Moreover, an intronic 7146G/A polymorphisms within the PD-1 gene, which result in reduced inhibitory function of PD-1 on cytokine production and T-cell activation, are associated with a progressive disease course in MS patients (Kroner et al., 2005). These findings demonstrate a potential role of PD1/PD-L1 in slowing the progression of MS. Consistent with these human studies, animal experiments in the EAE model of MS have shown that genetic ablation or pharmacological blockade of PD-1 or PD-L1 enhances the activation and expansion of T-cells and aggravates pathological alterations in the CNS (Latchman et al., 2001; Salama et al., 2003). In contrast, PD-L2 knockout mice develop similar pathologies as wild-type mice with no significant difference in severity (Carter et al., 2007). Furthermore, PD-L2 on microglial and CNS infiltrating APCs has been shown to be less potent than PD-L1 in the regulation of cytokine (IFN- $\gamma$, IL17 , etc.) production and the activation of auto-reactive T-cells (Schreiner et al., 2008). A study of PD-L1 or PD-L2 blockade in several mouse strains further suggests that differential effects of these two PD-L isoforms on the susceptibility and progression of EAE may be attributed to differences in genetic background (Zhu et al., 2006).

Mechanistic studies suggest that PD-1/PD-L signaling actively modulates the onset and progressive course of MS via the regulation of various types of immune cells, such as effector T-cells, DCs, Tregs, and NK T-cells (Latchman et al., 2004; Chang et al., 2008; Schreiner et al., 2008; Brandl et al., 2010). These mechanisms have been recently reviewed elsewhere (Joller et al., 2012) and are therefore not discussed further here. Due to the importance of the PD-1/PD-L system in MS, therapeutic strategies targeting PD$1 / \mathrm{PD}-\mathrm{L} 1$ interactions can be envisioned as an immunosuppressive treatment for MS patients. For example, estrogen has been shown to effectively protect against EAE through upregulating PD-L1 expression on B cells and increase the amount of IL-10-producing regulatory B cells (Bodhankar et al., 2011). It also induces B-celldependent up-regulation of PD- 1 on $\mathrm{CD} 4^{+}$Foxp $3^{+}$Tregs, which provide further protection against EAE (Bodhankar et al., 2012). IL-12, a cytokine mainly produced by APCs, is also shown to suppress the development of EAE through stimulating IFN- $\gamma$ production in APCs and enhancing downstream PD-1/PD-L1 signaling (Cheng et al., 2007). Further studies are warranted to assess the effectiveness of PD-1/PD-L1 modulation as a therapeutic strategy in MS patients.

\section{CONCLUSION}

The expression of PD-1 and PD-L on many immune and nonimmune cells surely allows for multiple tiers of immunoregulation in the CNS and remains an active area of investigation. Increasing numbers of clinical and experimental studies have shed light on the critical role of the PD-1/PD-L1 system in 
the regulation of resident microglia in the CNS and peripheral immune cells after brain injury and neurodegeneration. However, given the complexity of inflammatory responses in the CNS, our current understanding of the function of PD-1/PD$\mathrm{L}$ in the cross-talk between peripheral immune cells, CNS glial cells, and non-immune CNS cells still lies in its infancy. As an example, the expression of PD-L1/PD-L2 is up-regulated in inflamed endothelial cells, with an intention to inhibit T-cell transmigration through BBB (Pittet et al., 2011). In particular, the impaired expression of PD-L2 on endothelial cells may contribute to the cerebral inflammation in MS patients. The molecular mechanism underlying the PD-L2-afforded BBB resistance to T-cell infiltration, however, is not clear. Similarly, the expression of PD-L on astrocytes has been reported in a model of nerve injury (Lipp et al., 2007), however, whether and how astrocytic PD-L plays a role in restricting local inflammation in CNS has not been examined. It will also be important to determine whether modulation of PD-1/PD-L signaling pathway during CNS injury or neurodegeneration influence the balance between debris clearance, brain repair, and inflammatory damage. Further investigations of the PD-1/PD-L pathway in CNS disorders are warranted to improve our understanding of the mechanisms underlying immunomodulation and to develop effective and safe immunotherapies.

\section{ACKNOWLEDGMENTS}

Dr. Xiaoming Hu is supported by the American Heart Association (13SDG14570025) and the Ethyl Vincent pilot grant in multiple sclerosis from Department of Neurology, University of Pittsburgh. Dr. Rehana K. Leak is supported by a Commonwealth Universal Research Enhancement (C.U.R.E.) Award from the Department of Health and a Michael J. Fox Foundation Innovation Award. Dr. Jun Chen is supported by the National Institutes of Health Grants (NS45048, NS62157, NS59806, and NS36736), Chinese Natural Science Foundation grants (81228008), and a VA Merit Review.

\section{REFERENCES}

Asahi, M., Wang, X., Mori, T., Sumii, T., Jung, J. C., Moskowitz, M. A., et al. (2001). Effects of matrix metalloproteinase- 9 gene knock-out on the proteolysis of blood-brain barrier and white matter components after cerebral ischemia. J. Neurosci. 21, 7724-7732.

Blank, C., Brown, I., Marks, R., Nishimura, H., Honjo, T., and Gajewski, T. F. (2003). Absence of programmed death receptor 1 alters thymic development and enhances generation of CD4/CD8 double-negative TCR-transgenic T cells. J. Immunol. 171, 4574-4581. doi:10.4049/jimmunol.171.9.4574

Blocki, F. A., Radhakrishnan, S., Van Keulen, V. P., Heckman, K. L., Ciric, B., Howe, C. L., et al. (2006). Induction of a gene expression program in dendritic cells with a cross-linking IgM antibody to the co-stimulatory molecule B7-DC. FASEB J. 20, 2408-2410. doi:10.1096/fj.06-6171fje

Bodhankar, S., Chen, Y., Vandenbark, A. A., Murphy, S. J., and Offner, H. (2013a). IL-10-producing B-cells limit CNS inflammation and infarct volume in experimental stroke. Metab. Brain Dis. 28, 375-386. doi:10.1007/s11011-013-9413-3

Bodhankar, S., Chen, Y., Vandenbark, A. A., Murphy, S. J., and Offner, H. (2013b). PD-L1 enhances CNS inflammation and infarct volume following experimental stroke in mice in opposition to PD-1. J. Neuroinflammation 10, 111. doi:10.1186/1742-2094-10-111

Bodhankar, S., Vandenbark, A. A., and Offner, H. (2012). Oestrogen treatment of experimental autoimmune encephalomyelitis requires 17beta-oestradiolreceptor-positive $\mathrm{B}$ cells that up-regulate $\mathrm{PD}-1$ on $\mathrm{CD} 4+$ Foxp3+ regulatory $\mathrm{T}$ cells. Immunology 137, 282-293. doi:10.1111/imm.12013
Bodhankar, S., Wang, C., Vandenbark, A. A., and Offner, H. (2011). Estrogen-induced protection against experimental autoimmune encephalomyelitis is abrogated in the absence of B cells. Eur. J. Immunol. 41, 1165-1175. doi:10.1002/eji.201040992

Brandl, C., Ortler, S., Herrmann, T., Cardell, S., Lutz, M. B., and Wiendl, H. (2010). B7-H1-deficiency enhances the potential of tolerogenic dendritic cells by activating CD1d-restricted type II NKT cells. PLoS ONE 5:e10800. doi:10.1371/journal. pone. 0010800

Carter, L. L., Leach, M. W., Azoitei, M. L., Cui, J., Pelker, J. W., Jussif, J., et al. (2007). PD-1/PD-L1, but not PD-1/PD-L2, interactions regulate the severity of experimental autoimmune encephalomyelitis. J. Neuroimmunol. 182, 124-134. doi:10.1016/j.jneuroim.2006.10.006

Chang, W. S., Kim, J. Y., Kim, Y. J., Kim, Y. S., Lee, J. M., Azuma, M., et al. (2008). Cutting edge: programmed death-1/programmed death ligand 1 interaction regulates the induction and maintenance of invariant NKT cell anergy. J. Immunol. 181, 6707-6710. doi:10.4049/jimmunol.181.10.6707

Cheng, X., Zhao, Z., Ventura, E., Gran, B., Shindler, K. S., and Rostami, A. (2007). The PD-1/PD-L pathway is up-regulated during IL-12-induced suppression of EAE mediated by IFN-gamma. J. Neuroimmunol. 185, 75-86. doi:10.1016/j.jneuroim.2007.01.012

Compston, A., and Coles, A. (2008). Multiple sclerosis. Lancet 372, 1502-1517. doi:10.1016/S0140-6736(08)61620-7

Engelhardt, B. (2008). The blood-central nervous system barriers actively control immune cell entry into the central nervous system. Curr. Pharm. Des. 14, 1555-1565. doi:10.2174/138161208784705432

Fife, B. T., Pauken, K. E., Eagar, T. N., Obu, T., Wu, J., Tang, Q., et al. (2009). Interactions between PD-1 and PD-L1 promote tolerance by blocking the TCR-induced stop signal. Nat. Immunol. 10, 1185-1192. doi:10.1038/ni.1790

Freeman, G. J., Long, A. J., Iwai, Y., Bourque, K., Chernova, T., Nishimura, H., et al. (2000). Engagement of the PD-1 immunoinhibitory receptor by a novel B7 family member leads to negative regulation of lymphocyte activation. J. Exp. Med. 192, 1027-1034. doi:10.1084/jem.192.7.1027

Gelderblom, M., Leypoldt, F., Steinbach, K., Behrens, D., Choe, C. U., Siler, D. A., et al. (2009). Temporal and spatial dynamics of cerebral immune cell accumulation in stroke. Stroke 40, 1849-1857. doi:10.1161/STROKEAHA.108.534503

Ghiotto, M., Gauthier, L., Serriari, N., Pastor, S., Truneh, A., Nunes, J. A., et al. (2010). PD-L1 and PD-L2 differ in their molecular mechanisms of interaction with PD-1. Int. Immunol. 22, 651-660. doi:10.1093/intimm/dxq049

Guerreiro, R. J., Santana, I., Bras, J. M., Santiago, B., Paiva, A., and Oliveira, C. (2007). Peripheral inflammatory cytokines as biomarkers in Alzheimer's disease and mild cognitive impairment. Neurodegener. Dis. 4, 406-412. doi:10.1159/000107700

Iadecola, C., and Anrather, J. (2011). The immunology of stroke: from mechanisms to translation. Nat. Med. 17, 796-808. doi:10.1038/nm.2399

Joller, N., Peters, A., Anderson, A. C., and Kuchroo, V. K. (2012). Immune checkpoints in central nervous system autoimmunity. Immunol. Rev. 248, 122-139. doi:10.1111/j.1600-065X.2012.01136.x

Keir, M. E., Latchman, Y. E., Freeman, G. J., and Sharpe, A. H. (2005). Programmed death-1 (PD-1):PD-ligand 1 interactions inhibit TCR-mediated positive selection of thymocytes. J. Immunol. 175, 7372-7379. doi:10.4049/jimmunol. 175.11 .7372

Koronyo-Hamaoui, M., Ko, M. K., Koronyo, Y., Azoulay, D., Seksenyan, A., Kunis, G., et al. (2009). Attenuation of AD-like neuropathology by harnessing peripheral immune cells: local elevation of IL-10 and MMP-9. J. Neurochem. 111, 1409-1424. doi:10.1111/j.1471-4159.2009.06402.x

Kroner, A., Mehling, M., Hemmer, B., Rieckmann, P., Toyka, K. V., Maurer, M., et al. (2005). A PD-1 polymorphism is associated with disease progression in multiple sclerosis. Ann. Neurol. 58, 50-57. doi:10.1002/ana.20514

Langrish, C. L., Chen, Y., Blumenschein, W. M., Mattson, J., Basham, B., Sedgwick, J. D., et al. (2005). IL-23 drives a pathogenic T cell population that induces autoimmune inflammation. J. Exp. Med. 201, 233-240. doi:10.1084/jem.20041257

Latchman, Y., Wood, C. R., Chernova, T., Chaudhary, D., Borde, M., Chernova, I., et al. (2001). PD-L2 is a second ligand for PD-1 and inhibits T cell activation. Nat. Immunol. 2, 261-268. doi:10.1038/85330

Latchman, Y. E., Liang, S. C., Wu, Y., Chernova, T., Sobel, R. A., Klemm, M., et al. (2004). PD-L1-deficient mice show that PD-L1 on T cells, antigen-presenting cells, and host tissues negatively regulates T cells. Proc. Natl. Acad. Sci. U.S.A. 101, 10691-10696. doi:10.1073/pnas.0307252101

Lee, Y., Moon, Y., Hyung, K., Yoo, J., Lee, M., Lee, I., et al. (2013). Macrophage PDL1 strikes back: PD-1/PD-L1 interaction drives macrophages toward regulatory subsets. Adv. Biosci. Biotechnol. 4, 19-29. doi:10.4236/abb.2013.48A3003 
Li, P., Gan, Y., Sun, B. L., Zhang, F., Lu, B., Gao, Y., et al. (2013). Adoptive regulatory T-cell therapy protects against cerebral ischemia. Ann. Neurol. 74, 458-471. doi:10.1002/ana.23815

Li, P., Mao, L., Liu, X., Gan, Y., Zheng, J., Thomson, A. W., et al. (2014). Essential role of program death 1-ligand 1 in regulatory T-cell-afforded protection against blood-brain barrier damage after stroke. Stroke 45, 857-864. doi:10.1161/STROKEAHA.113.004100

Liesz, A., Suri-Payer, E., Veltkamp, C., Doerr, H., Sommer, C., Rivest, S., et al. (2009). Regulatory $\mathrm{T}$ cells are key cerebroprotective immunomodulators in acute experimental stroke. Nat. Med. 15, 192-199. doi:10.1038/nm.1927

Liesz, A., Zhou, W., Mracsko, E., Karcher, S., Bauer, H., Schwarting, S., et al. (2011). Inhibition of lymphocyte trafficking shields the brain against deleterious neuroinflammation after stroke. Brain 134, 704-720. doi:10.1093/brain/awr008

Lipp, M., Brandt, C., Dehghani, F., Kwidzinski, E., and Bechmann, I. (2007). PD-L1 (B7-H1) regulation in zones of axonal degeneration. Neurosci. Lett. 425, 156-161. doi:10.1016/j.neulet.2007.07.053

Liu, Y. H., Zeng, F., Wang, Y. R., Zhou, H. D., Giunta, B., Tan, J., et al. (2013). Immunity and Alzheimer's disease: immunological perspectives on the development of novel therapies. Drug Discov. Today 18, 1212-1220. doi:10.1016/j.drudis.2013. 07.020

Minagar, A., Toledo, E. G., Alexander, J. S., and Kelley, R. E. (2004). Pathogenesis of brain and spinal cord atrophy in multiple sclerosis. J. Neuroimaging 14, 5S-10S. doi:10.1111/j.1552-6569.2004.tb00273.x

Nishimura, H., Nose, M., Hiai, H., Minato, N., and Honjo, T. (1999). Development of lupus-like autoimmune diseases by disruption of the PD-1 gene encoding an ITIM motif-carrying immunoreceptor. Immunity 11, 141-151. doi:10.1016/S1074-7613(00)80089-8

Nishimura, H., Okazaki, T., Tanaka, Y., Nakatani, K., Hara, M., Matsumori, A., et al. (2001). Autoimmune dilated cardiomyopathy in PD-1 receptor-deficient mice. Science 291, 319-322. doi:10.1126/science.291.5502.319

Okazaki, T., Chikuma, S., Iwai, Y., Fagarasan, S., and Honjo, T. (2013). A rheostat for immune responses: the unique properties of PD-1 and their advantages for clinical application. Nat. Immunol. 14, 1212-1218. doi:10.1038/ni.2762

Okazaki, T., Maeda, A., Nishimura, H., Kurosaki, T., and Honjo, T. (2001). PD-1 immunoreceptor inhibits B cell receptor-mediated signaling by recruiting src homology 2-domain-containing tyrosine phosphatase 2 to phosphotyrosine. Proc. Natl. Acad. Sci. U.S.A. 98, 13866-13871. doi:10.1073/pnas.231486598

Pittet, C. L., Newcombe, J., Prat, A., and Arbour, N. (2011). Human brain endothelial cells endeavor to immunoregulate CD8 T cells via PD-1 ligand expression in multiple sclerosis. J. Neuroinflammation 8, 155. doi:10.1186/17422094-8-155

Probst, H. C., Mccoy, K., Okazaki, T., Honjo, T., and Van Den Broek, M. (2005). Resting dendritic cells induce peripheral CD8+ T cell tolerance through PD-1 and CTLA-4. Nat. Immunol. 6, 280-286. doi:10.1038/ni1165

Ren, X., Akiyoshi, K., Dziennis, S., Vandenbark, A. A., Herson, P. S., Hurn, P. D., et al. (2011a). Regulatory B cells limit CNS inflammation and neurologic deficits in murine experimental stroke. J. Neurosci. 31, 8556-8563. doi:10.1523/JNEUROSCI.1623-11.2011

Ren, X., Akiyoshi, K., Vandenbark, A. A., Hurn, P. D., and Offner, H. (2011b). Programmed death-1 pathway limits central nervous system inflammation and neurologic deficits in murine experimental stroke. Stroke 42, 2578-2583. doi:10.1161/STROKEAHA.111.613182

Reynoso, E. D., Elpek, K. G., Francisco, L., Bronson, R., Bellemare-Pelletier, A., Sharpe, A. H., et al. (2009). Intestinal tolerance is converted to autoimmune enteritis upon PD-1 ligand blockade. J. Immunol. 182, 2102-2112. doi:10.4049/ jimmunol.0802769

Rifa'i, M., Kawamoto, Y., Nakashima, I., and Suzuki, H. (2004). Essential roles of CD8+CD122+ regulatory T cells in the maintenance of T cell homeostasis. J. Exp. Med. 200, 1123-1134. doi:10.1084/jem.20040395

Riley, J. L., and June, C. H. (2005). The CD28 family: a T-cell rheostat for therapeutic control of T-cell activation. Blood 105, 13-21. doi:10.1182/blood-2004-04-1596

Rosell, A., Ortega-Aznar, A., Alvarez-Sabin, J., Fernandez-Cadenas, I., Ribo, M., Molina, C. A., et al. (2006). Increased brain expression of matrix metalloproteinase-9 after ischemic and hemorrhagic human stroke. Stroke 37, 1399-1406. doi:10.1161/01.STR.0000223001.06264.af

Rubio-Perez, J. M., and Morillas-Ruiz, J. M. (2012). A review: inflammatory process in Alzheimer's disease, role of cytokines. ScientificWorldJournal 2012, 756357. doi: $10.1100 / 2012 / 756357$
Said, E. A., Dupuy, F. P., Trautmann, L., Zhang, Y., Shi, Y., El-Far, M., et al. (2010). Programmed death-1-induced interleukin-10 production by monocytes impairs CD4+ T cell activation during HIV infection. Nat. Med. 16, 452-459. doi:10.1038/nm.2106

Salama, A. D., Chitnis, T., Imitola, J., Ansari, M. J., Akiba, H., Tushima, F., et al. (2003). Critical role of the programmed death-1 (PD-1) pathway in regulation of experimental autoimmune encephalomyelitis. J. Exp. Med. 198, 71-78. doi:10.1084/jem.20022119

Saresella, M., Calabrese, E., Marventano, I., Piancone, F., Gatti, A., Calvo, M. G., et al. (2010). PD1 negative and PD1 positive CD4+ T regulatory cells in mild cognitive impairment and Alzheimer's disease. J. Alzheimers Dis. 21, 927-938. doi:10.3233/JAD-2010-091696

Saresella, M., Calabrese, E., Marventano, I., Piancone, F., Gatti, A., Farina, E., et al. (2012). A potential role for the PD1/PD-L1 pathway in the neuroinflammation of Alzheimer's disease. Neurobiol. Aging 33, e611-e622. doi:10.1016/j. neurobiolaging.2011.03.004

Schreiner, B., Bailey, S. L., Shin, T., Chen, L., and Miller, S. D. (2008). PD-1 ligands expressed on myeloid-derived APC in the CNS regulate T-cell responses in EAE. Eur. J. Immunol. 38, 2706-2717. doi:10.1002/eji.200838137

Sheppard, K. A., Fitz, L. J., Lee, J. M., Benander, C., George, J. A., Wooters, J., et al. (2004). PD-1 inhibits T-cell receptor induced phosphorylation of the ZAP70/CD3zeta signalosome and downstream signaling to PKCtheta. FEBS Lett. 574, 37-41. doi:10.1016/j.febslet.2004.07.083

Speciale, L., Calabrese, E., Saresella, M., Tinelli, C., Mariani, C., Sanvito, L., et al. (2007). Lymphocyte subset patterns and cytokine production in Alzheimer's disease patients. Neurobiol. Aging 28, 1163-1169. doi:10.1016/j.neurobiolaging. 2006.05.020

Torres, K. C., Araujo Pereira, P., Lima, G. S., Bozzi, I. C., Rezende, V. B., Bicalho, M. A., et al. (2013). Increased frequency of T cells expressing IL-10 in Alzheimer disease but not in late-onset depression patients. Prog. Neuropsychopharmacol. Biol. Psychiatry 47, 40-45. doi:10.1016/j.pnpbp.2013.07.021

Trabattoni, D., Saresella, M., Pacei, M., Marventano, I., Mendozzi, L., Rovaris, M., et al. (2009). Costimulatory pathways in multiple sclerosis: distinctive expression of PD-1 and PD-L1 in patients with different patterns of disease. J. Immunol. 183, 4984-4993. doi:10.4049/jimmunol.0901038

Tzartos, J. S., Friese, M. A., Craner, M. J., Palace, J., Newcombe, J., Esiri, M. M., et al. (2008). Interleukin-17 production in central nervous system-infiltrating T cells and glial cells is associated with active disease in multiple sclerosis. Am. J. Pathol. 172, 146-155. doi:10.2353/ajpath.2008.070690

Viglietta, V., Baecher-Allan, C., Weiner, H. L., and Hafler, D. A. (2004). Loss of functional suppression by CD4+CD25+ regulatory $\mathrm{T}$ cells in patients with multiple sclerosis. J. Exp. Med. 199, 971-979. doi:10.1084/jem.20031579

Wang, C., Li, Y., Proctor, T. M., Vandenbark, A. A., and Offner, H. (2010). Down-modulation of programmed death 1 alters regulatory $\mathrm{T}$ cells and promotes experimental autoimmune encephalomyelitis. J. Neurosci. Res. 88, 7-15. doi:10.1002/jnr.22181

Yilmaz, G., Arumugam, T. V., Stokes, K. Y., and Granger, D. N. (2006). Role of T lymphocytes and interferon-gamma in ischemic stroke. Circulation 113, 2105-2112. doi:10.1161/CIRCULATIONAHA.105.593046

Yokosuka, T., Takamatsu, M., Kobayashi-Imanishi, W., Hashimoto-Tane, A., Azuma, M., and Saito, T. (2012). Programmed cell death 1 forms negative costimulatory microclusters that directly inhibit $\mathrm{T}$ cell receptor signaling by recruiting phosphatase SHP2. J. Exp. Med. 209, 1201-1217. doi:10.1084/jem.20112741

Zhang, X., Schwartz, J. C., Guo, X., Bhatia, S., Cao, E., Lorenz, M., et al. (2004). Structural and functional analysis of the costimulatory receptor programmed death-1. Immunity 20, 337-347. doi:10.1016/S1074-7613(04)00051-2

Zhong, X., Tumang, J. R., Gao, W., Bai, C., and Rothstein, T. L. (2007). PD-L2 expression extends beyond dendritic cells/macrophages to $\mathrm{B} 1$ cells enriched for $\mathrm{V}(\mathrm{H}) 11 / \mathrm{V}(\mathrm{H}) 12$ and phosphatidylcholine binding. Eur. J. Immunol. 37, 2405-2410. doi:10.1002/eji.200737461

Zhu, B., Guleria, I., Khosroshahi, A., Chitnis, T., Imitola, J., Azuma, M., et al. (2006). Differential role of programmed death-ligand 1 [corrected] and programmed death-ligand 2 [corrected] in regulating the susceptibility and chronic progression of experimental autoimmune encephalomyelitis. J. Immunol. 176, 3480-3489. doi:10.4049/jimmunol.176.6.3480

Zipp, F., and Aktas, O. (2006). The brain as a target of inflammation: common pathways link inflammatory and neurodegenerative diseases. Trends Neurosci. 29, 518-527. doi:10.1016/j.tins.2006.07.006 
Conflict of Interest Statement: The authors declare that the research was conducted in the absence of any commercial or financial relationships that could be construed as a potential conflict of interest.

Received: 29 July 2014; accepted: 20 August 2014; published online: 03 September 2014. Citation: Zhao S, Li F, Leak RK, Chen J and Hu X (2014) Regulation of neuroinflammation through programed death-1/programed death ligand signaling in neurological disorders. Front. Cell. Neurosci. 8:271. doi: 10.3389/fncel.2014.00271
This article was submitted to the journal Frontiers in Cellular Neuroscience. Copyright (c) 2014 Zhao, Li, Leak, Chen and Hu. This is an open-access article distributed under the terms of the Creative Commons Attribution License (CC BY). The use, distribution or reproduction in other forums is permitted, provided the original author(s) or licensor are credited and that the original publication in this journal is cited, in accordance with accepted academic practice. No use, distribution or reproduction is permitted which does not comply with these terms. 